\title{
PROCEEDINGS
}

OF THE

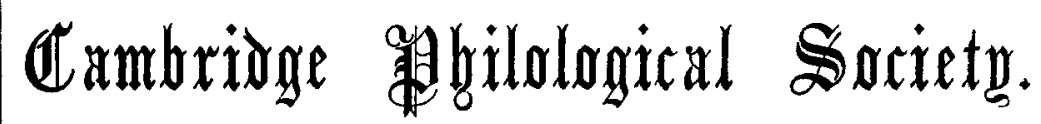

CLXVI-CLXVIII.

LENT, EASTER AND MICHAELMAS

TERMS, 1937

CAMBRIDGE UNIVERSITY PRESS

LONDON

1938

(All rights reserved)

Price One Shilling net. 


\title{
Cambrituge Philologital Soctety
}

\section{COUNCIL FOR 1937}

\author{
3regtoent
}

Pror. A. J. B. WACE, M.A., Pembroke

\section{Fice-Fresioents}

R. HACKFORTH, M.A., Sidney Sussex Prof. D. S. ROBERTSON, M.A., Trinity

The Provost of King's Colleae

\section{Oroinarn flembers of Council}

R. G. BURY, Litr.D., Thrnity

D. W. LUCAS, M.A., KINe's

A. P. SINKER, M.A., JESDB

Prof. F. E. ADCOCK, M.A., Krng's

C. T. SELTMAN, M.A., QUEEN'

Mrss J. M. C. TOYNBEE, M.A., Newn.

M. P. CHARLESWORTH, M.A.,
R. J. H. JENKINS, M.A., Emancel

A. S. F. GOW, M.A., Trinity

Prof. W. B. ANDERSON, LITT.D.,

ST JoHN's

R. L. HOWLAND, M.A., ST JoHN's

W. K. C. GUTHRIE, M.A.,

Peterhodse

7\%on. Treasurer

H. T. DEAS, M.A., CaIUs

\section{F⿻日土 Son. Setretaries}

R. M. RATtenbURY, M.A., Trinty | N. G. L. Hammond, M.A., Clare

Fon. Ilibrarian

B. L. HallWard, M.A., Petrergouse

79on. Audtors

J. M. EDMONDS, M.A., JHSUs

B. G. CAMPBELL, M.A., Crars's's

Wankets: BARCLAYS BANK LTD., Cambrider 